\title{
An Estimate of the Maximum Gradients in Superconducting Quadrupoles
}

\author{
Ezio Todesco and Lucio Rossi
}

\begin{abstract}
In this paper we show that the electromagnetic design of several superconducting quadrupoles, built for particle accelerators, can be rather well approximated by a 36 degree sector coil with a wedge, canceling the first two field harmonics. We therefore carry out a complete analysis of this lay-out, obtaining an approximated equation for the critical gradient as a function of the coil area, magnet aperture, and of the superconducting properties of the cable. Using this model, we estimate through numerical methods the maximum critical gradient that can be obtained in quadrupole of a given aperture for $\mathrm{Nb}-\mathrm{Ti}, \mathrm{Nb}-\mathrm{Ti}-\mathrm{Ta}$ and $\mathrm{Nb}_{3} \mathrm{Sn}$.
\end{abstract}

Index Terms-Accelerator magnets, superconducting magnets.

\section{INTRODUCTION}

$\mathbf{S}$ UPERCONDUCTING quadrupoles have been used to focus particle beams in accelerators and storage rings since more than 30 years [1]-[3]. A simple upper bound to the maximum achievable gradient is given by the following condition: the gradient times the aperture radius cannot exceed the critical field of the superconducting material, which is $\sim 13 \mathrm{~T}$ for the $\mathrm{Nb}-\mathrm{Ti}$, and $\sim 25 \mathrm{~T}$ for the $\mathrm{Nb}_{3} \mathrm{Sn}$, both at $1.9 \mathrm{~K}$. Indeed, the critical field is associated to a vanishing current density and therefore this naïve limit can be considered as academic.

In this paper we aim at giving a better estimate of the maximum gradient achievable in an accelerator superconducting quadrupole. Following a similar approach to [4]-[6], we analyze a simplified coil lay-out to derive an equation for the gradient as a function of the quadrupole aperture, of the quantity of cable in the cross-section, and on the superconducting properties of the cable. Instead of choosing the classical $\cos 2 \theta$ lay-out, we focus on a $36^{\circ}$ sector coil with a wedge to zero the first two field harmonics (see Fig. 1), which is much closer to the structure of an actual coil built with cable [7]. We apply this formula to several built quadrupoles to verify if our model well represents realistic lay-outs. We then use this equation to derive an estimate of the maximum achievable gradient.

\section{LAY-OUT PARAMETERS OF AN IRONLESS SECTOR COIL}

We consider a $36^{\circ}$ quadrupole sector coil with a wedge between $24^{\circ}$ and $30^{\circ}$ (see Fig. 1), with aperture radius $r$ and coil

Manuscript received August 28, 2006. This work was supported by the European Community-Research Infrastructure Activity under the FP6 "Structuring the European Research Area" Program (CARE, Contract RII3-CT-2003-506395).

The authors are with the Accelerator Technology Department, CERN, 1211 Geneva 23, Switzerland (e-mail: ezio.todesco@cern.ch).

Color versions of one or more of the figures in this paper are available online at http://ieeexplore.ieee.org.

Digital Object Identifier 10.1109/TASC.2007.898428

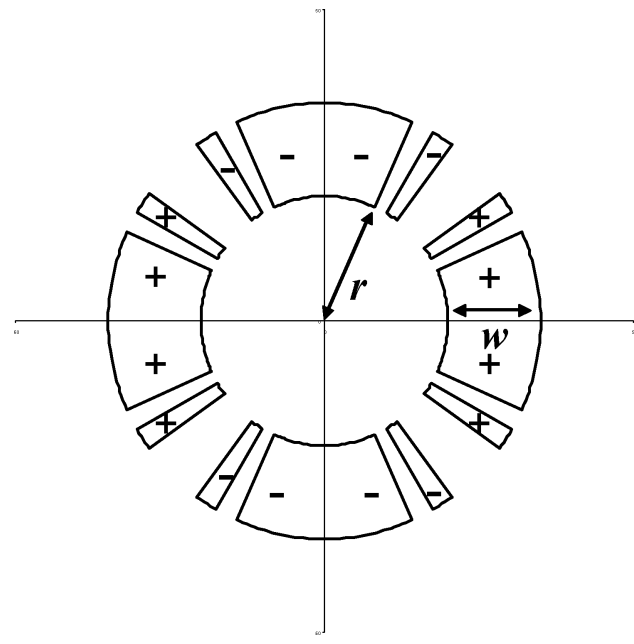

Fig. 1. Layout of a $36^{\circ}$ sector coil with a wedge between $24^{\circ}$ and $30^{\circ}$ for a quadrupole of aperture radius $r$ and coil width $w$.

width $w$. This lay-out cancels the first two field harmonics $b_{6}$ and $b_{10}$. We assume that there is no iron contributing to the field, and that the current density $j\left[\mathrm{~A} / \mathrm{m}^{2}\right]$ is uniform.

Both the field gradient $G[\mathrm{~T} / \mathrm{m}]$ at the centre of the quadrupole and the peak field $B_{p}$ [T], i.e. the largest value (in module) of the magnetic field in the coil, are proportional to the current density $j$ :

$$
\begin{aligned}
G & =j \gamma, \\
B_{p} & =j \beta .
\end{aligned}
$$

We compute $\gamma[\mathrm{Tm} / \mathrm{A}]$ through a simple integration of the BiotSavart law

$$
\gamma=\gamma_{0} \log \left(1+\frac{w}{r}\right)
$$

where

$$
\gamma_{0}=\frac{4}{5} 10^{-6}\left(\sin \frac{6 \pi}{15}-\sin \frac{\pi}{3}+\sin \frac{4 \pi}{15}\right) \approx 0.663 \cdot 10^{-6} \mathrm{Tm} / \mathrm{A}
$$

and we write the parameter $\beta\left[\mathrm{Tm}^{2} / \mathrm{A}\right]$ in the form

$$
\beta=r \lambda \gamma,
$$

where $\gamma$ [adimensional] is the ratio between the peak field and the gradient times the aperture. The parameter $\gamma$ is equivalent to the ratio between the peak field and the central field in a dipole magnet. The dependence of $\gamma$ on $w / r$ has been numerically evaluated, finding out (see Fig. 2) that it can be well fit by

$$
\lambda \sim a_{-1} \frac{r}{w}+1+a_{1} \frac{w}{r},
$$




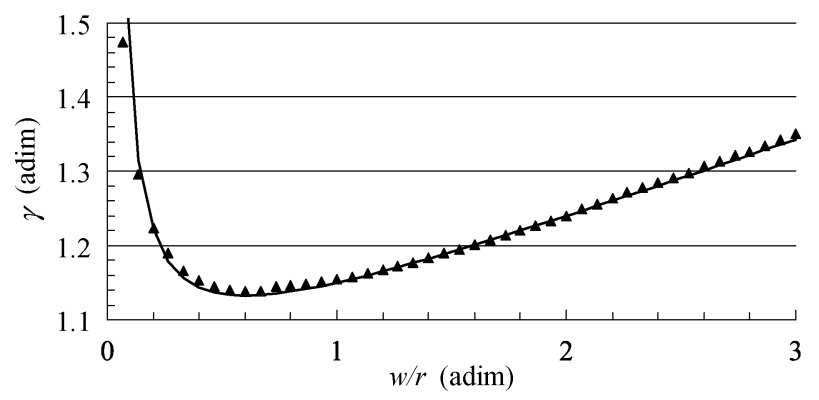

Fig. 2. Parameter $\lambda$ defined in (5) versus $w / r$ : numerical evaluation (markers) and analytical fit of (6).

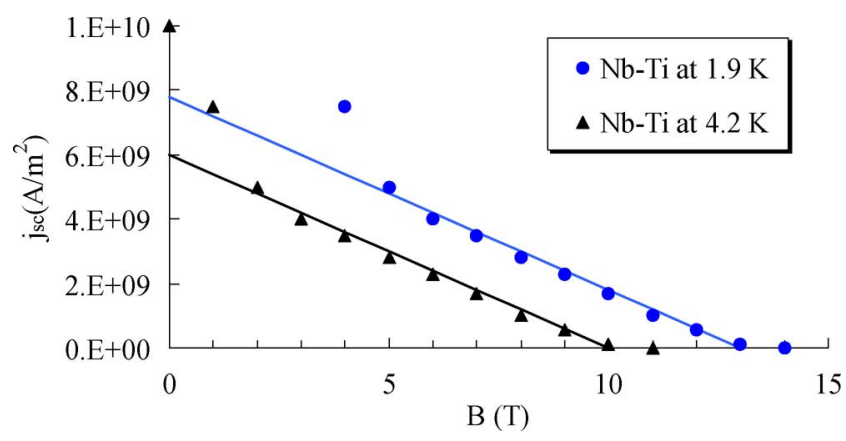

Fig. 3. Critical surface for $\mathrm{Nb}-\mathrm{Ti}$ at $1.9 \mathrm{~K}$ and at $4.4 \mathrm{~K}$, and linear fit given in (1), data relative to LHC superconductors.

with $a_{-1}=0.042$ and $a_{1}=0.113$. For values of $w / r$ between $1 / 3$ and 1 , which are typical of most magnet lay-outs (see Section III-B), $\gamma$ is around 1.15 , i.e. the peak field is $15 \%$ larger than the gradient times the aperture radius. For larger $w / r$ the ratio $\lambda$ increases proportionally to $w / r$.

\section{CRITICAL GRADIENT FOR Nb-Ti}

\section{A. Critical Gradient of an Ironless Sector Coil}

A $\mathrm{Nb}$-Ti filament carrying a current density $j_{\mathrm{sc}}$ in a magnetic field $B$ is superconducting as long as $j_{\mathrm{sc}}$ is less than the critical current density $j_{\mathrm{sc}, c}$, which can be approximated by a linear function of the magnetic field:

$$
j_{s c, c}=c\left(B_{c 2}^{*}-B\right), \quad B<B_{c 2}^{*}
$$

where $B_{c 2}^{*}$ is the critical field at zero current according to the standard linear fit, that underestimates the actual critical field value of around $10 \%$ (see Fig. 3), and $c\left[\mathrm{~A} /\left(\mathrm{T} \cdot \mathrm{m}^{2}\right)\right]$ is the slope of the line in the $\left(j_{\mathrm{sc}}, B\right)$ plane. The fit is good for $\mathrm{B} \geq 5 \mathrm{~T}$ at $1.9 \mathrm{~K}$, and $\mathrm{B} \geq 2 \mathrm{~T}$ at $4.2 \mathrm{~K}$, which is the interesting domain for our analysis. One has $B_{c 2}^{*} \sim 10 \mathrm{~T}$ at $4.2 \mathrm{~K}$ and $\sim 13 \mathrm{~T}$ at 1.9 $\mathrm{K}$, whereas the slope $c \sim 6 \times 10^{8} \mathrm{~A} /\left(\mathrm{T} \cdot \mathrm{m}^{2}\right)$ is independent of the temperature. This corresponds to having $3 \times 10^{9} \mathrm{~A} / \mathrm{m}^{2}$ at 8 $\mathrm{T}$ and $1.9 \mathrm{~K}$, or at $5 \mathrm{~T}$ and $4.2 \mathrm{~K}$.

A practical cable is made of an insulated conductor, composed of wires, made of $\mathrm{Nb}$-Ti filaments in a copper matrix. One

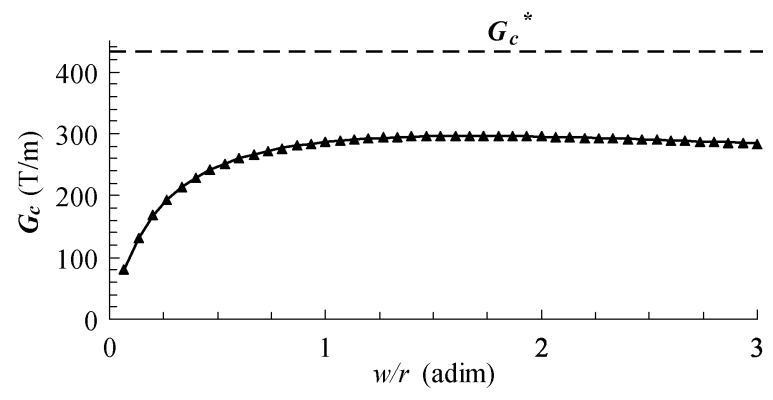

Fig. 4. Critical gradient versus sector width for the $\left[0-24,30^{\circ}-36\right]$ sector: numerical results (markers) and analytical approximation by (11) (solid line), aperture radius $r=30 \mathrm{~mm}$ and filling factor $\kappa=0.25$.

defines an engineering current density $j$, i.e. the current density flowing in the insulated conductor

$$
j=\kappa_{w-c} \kappa_{c-i} \frac{j_{s c}}{1+\nu_{C u-s c}}=\kappa j_{s c}
$$

where we defined the filling factor $\kappa$ that depends on i) the ratio $\kappa_{\mathrm{w}-\mathrm{c}}$ between the area of the strands in the conductor and the area of the bare conductor, ii) on the ratio $\kappa_{\mathrm{c}-\mathrm{i}}$ between the area of the bare conductor and of the insulated conductor, and iii) on the volume ratio $\nu_{\mathrm{Cu}-\mathrm{sc}}$ between copper and superconductor in the strands. Typical values are $0.85-0.9$ for both $\kappa_{\mathrm{w}-\mathrm{c}}$ and $\nu_{\mathrm{c}-\mathrm{i}}$, and 1 to 2 for $\nu_{\mathrm{Cu}-\mathrm{sc}}$, thus giving $\kappa$ in a range between 0.25 and 0.35 .

The fit for the critical current surface can then be written as

$$
j_{c}=\kappa c\left(B_{c 2}^{*}-B\right) .
$$

Using the definitions (1) and (2) of $\gamma$ and $\beta$, one obtains the critical gradient (also called short sample gradient [5], [6]), defined as the gradient corresponding to a current density on the critical surface

$$
G_{c}=\frac{\kappa c B_{c 2}^{*}}{1+\kappa c \beta} \gamma=\frac{\kappa c B_{c 2}^{*}}{1+\kappa c r \gamma \lambda} \gamma
$$

Substituting the expressions ((3)-(6)) for $\gamma$ and $\beta$ one obtains

$$
G_{c} \sim \frac{\kappa c B_{c 2}^{*} \gamma_{0} \log \left(1+\frac{w}{r}\right)}{1+\kappa c r\left(a_{-1} \frac{r}{w}+1+a_{1} \frac{w}{r}\right) \gamma_{0} \log \left(1+\frac{w}{r}\right)},
$$

with $\gamma_{0}=0.66310^{-6} \mathrm{Tm} / \mathrm{A}$ as in (4), and $a_{-1}=0.042$ and $a_{1}=0.113$ are the adimensional constants defined in (6), the slope $c \sim 6 \times 10^{8} \mathrm{~A} /\left(\mathrm{T} \cdot \mathrm{m}^{2}\right), r$ and $w$ are expressed in meters, $B_{c 2}^{*}$ in $\mathrm{T}$, and the gradient in $\mathrm{T} / \mathrm{m}$.

According to this equation, for a given aperture radius $r$ the critical gradient strongly increases with $w$ for small $w / r$, then it saturates, and then slowly decreases for large $w / r$ (see Fig. 4). The somewhat unexpected fact that adding cable decreases the critical gradient is due to the asymptotic behaviors $\gamma \sim \log (w)$, whereas $\beta \sim w \log (w)$ : when adding cable for large $w$ we mainly increase the peak field, and we only marginally increase the gradient, thus reducing the critical gradient. This feature is typical of quadrupoles and is not found in dipoles [7]. In Fig. 4 we also plot the value $G_{\mathrm{c}}^{*} \equiv B_{c 2}^{*} / r$, that is the naive upper 
TABLE I

Parameters of 13 Superconducting Quadrupoles and Error of the Critical Gradient Estimate According to Eq. (14)

\begin{tabular}{ccccccc}
\hline Name & $\begin{array}{c}\text { Ap. radius } \\
(\mathrm{mm})\end{array}$ & $\begin{array}{c}\text { Layers, } \\
\text { blocks }\end{array}$ & $\begin{array}{c}\text { Filling } \\
\text { ratio }\end{array}$ & $\begin{array}{c}\text { aspect } \\
\text { ratio weq/r }\end{array}$ & $\begin{array}{c}\text { Grading } \\
(\%)\end{array}$ & $\begin{array}{c}\text { Difference } \\
(\%)\end{array}$ \\
\hline \hline ISR MQ & 116 & 1,3 & 0.35 & 0.28 & - & 2.7 \\
Tevatron MQ & 45 & 2,3 & 0.25 & 0.35 & - & -1.4 \\
HERA MQ & 37 & 2,3 & 0.27 & 0.49 & - & -0.6 \\
SSC MQ & 20 & 2,4 & 0.27 & 0.92 & - & 0.0 \\
LEP I MQC & 90 & 1,2 & 0.31 & 0.29 & - & 0.5 \\
LEP II MQC & 80 & 1,2 & 0.33 & 0.27 & - & -0.5 \\
RHIC MQ & 40 & 1,2 & 0.23 & 0.23 & - & 1.0 \\
RHIC MQY & 65 & 1,3 & 0.27 & 0.18 & - & 1.3 \\
LHC MQ & 28 & 2,4 & 0.25 & 1.01 & - & 3.6 \\
LHC MQM & 28 & 2,4 & 0.26 & 0.61 & - & 0.1 \\
LHC MQY & 35 & 4,5 & 0.34 & 0.79 & 43 & 8.2 \\
LHC MQXA & 35 & 4,6 & 0.34 & 1.08 & 10 & 3.1 \\
LHC MQXB & 35 & 2,4 & 0.33 & 0.76 & 27 & 5.6 \\
\hline
\end{tabular}

bound to the maximum critical gradient. We point out that in this case, one obtains at most only $70 \%$ of $G_{\mathrm{c}}^{*}$.

The agreement between the approximation (11) and a numerical computation is within $1 \%$ (see Fig. 4). To generalize (11) to any lay-out we express it in terms of the area of the insulated conductor. In our model one has

$$
A=\frac{2 \pi}{3}\left[(r+w)^{2}-r^{2}\right] \Rightarrow 1+\frac{w}{r}=\sqrt{1+\frac{3 A}{2 \pi r^{2}}},
$$

and therefore for any coil, whose surface is $A$, we can define an aspect ratio as

$$
\frac{w_{e q}}{r} \equiv \sqrt{1+\frac{3 A}{2 \pi r^{2}}}-1
$$

and we can express (11) in terms of the coil area $A$

$$
G_{c} \sim \frac{\kappa c B_{c 2}^{*} \gamma_{0} \log \left(1+\frac{w_{e q}}{r}\right)}{1+\kappa c r\left(a_{-1} \frac{r}{w_{e q}}+1+a_{1} \frac{w_{e q}}{r}\right) \gamma_{0} \log \left(1+\frac{w_{e q}}{r}\right)} .
$$

A simplified expression of the above equation with $\lambda \sim \lambda_{0}=$ 1.165 can also be defined, and gives good results for $w / r$ between $1 / 3$ and 1 , which is the usual range of interest of magnet designers. Indeed, this approximation does not account of the slow decay for large $w / r$, and does not provide a correct estimate of the maximum critical gradient.

\section{B. Comparison With Built Magnets}

We tested our estimate (14) for 13 quadrupoles, based on a shell design built in the last 30 years, with apertures radii from 20 to $116 \mathrm{~mm}, 1$ to 4 layers, and 2 to 6 blocks. Parameters are listed in Table I, where also the aspect ratio, defined using (13), is given. The comparison (last column of the Table) is made between the critical gradient estimated with $r, A, \kappa$ through (14), and a numerical computation for the actual lay-out without iron. The error is always below 3.5\%, with the exception of three cases, where current grading is used, which can provide up to $8 \%$ more critical gradient.

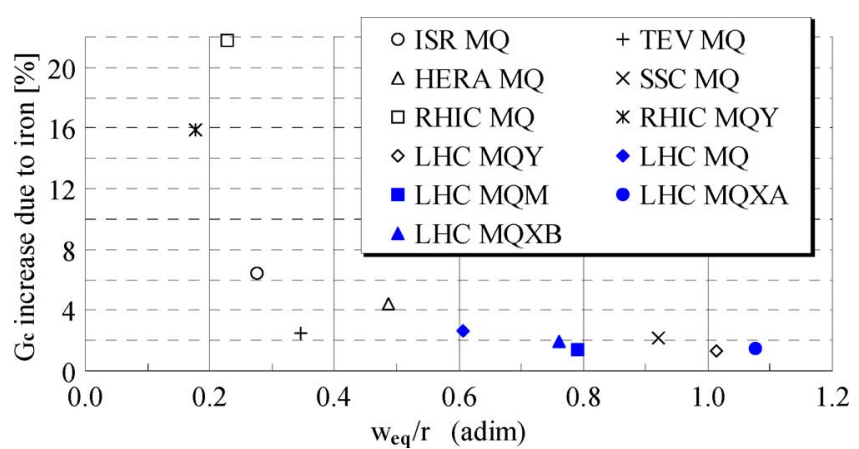

Fig. 5. Relative increase in the critical gradient due to the iron in 11 built quadrupoles.

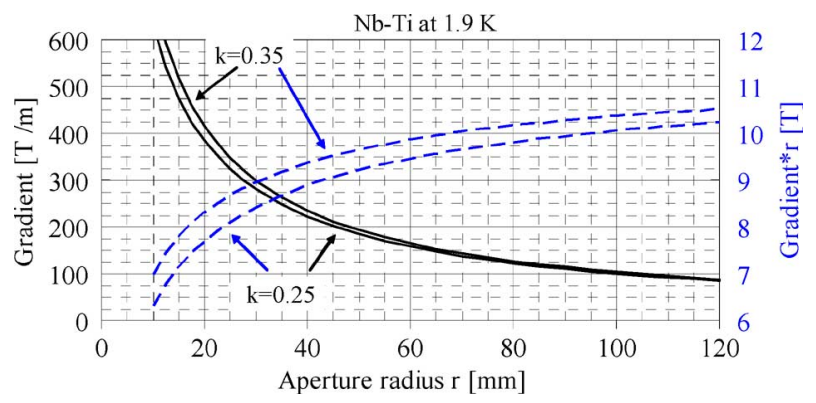

Fig. 6. Maximum critical gradient (solid line) and maximum critical gradient times aperture (dashed line) versus aperture radius for a sector coil of $\mathrm{Nb}-\mathrm{Ti}$ at $1.9 \mathrm{~K}$ for two different filling factor values.

\section{Effect of Iron}

The presence of iron has the main function of closing the magnetic circuit, and can be used to withstand the forces (mechanical function). The iron induces a higher field in the magnet aperture for the same current density. However, it also induces a higher peak field and therefore the beneficial effect on the critical gradient it is much less than what could be expected at a first view. Here, we computed the relative increase of the critical gradient for the coil layouts analyzed in the previous section. One finds that the magnets with lower $w / r$ (RHIC and ISR) have a non negligible contribution of the iron (7\% to $22 \%$ ), and for an aspect ratio larger than 0.5 the iron contribution is nearly negligible (1.5\% to $3 \%)$. Therefore we conclude that the iron does not affect the maximum critical gradient that can be reached for a given coil aperture, usually obtained with $w / r \sim 1$ (see Fig. 5).

\section{Estimating the Maximum Critical Gradient}

We compute the maximum critical gradient reachable for a given aperture as the maximum of (14) over $w_{\text {eq }}$. Results are shown in Fig. 6, where we also plot the gradient times the aperture radius that, according to the naïve estimate, should be $13 \mathrm{~T}$ for $\mathrm{Nb}-\mathrm{Ti}$ at $1.9 \mathrm{~K}$. One observes that for small apertures one obtains much less, namely $7 \mathrm{~T}$ for $r=10 \mathrm{~mm}$, and $9 \mathrm{~T}$ for $25 \mathrm{~mm}$. The upper bound of $13 \mathrm{~T}$ is never reached, since according to our model the peak field is always at least $15 \%$ larger than $r G$. This feature is also shared by the analyzed magnet lay-outs. The dependence on the filling factor is rather weak, and becomes relevant $(>5 \%)$ for $r<20 \mathrm{~mm}$. 


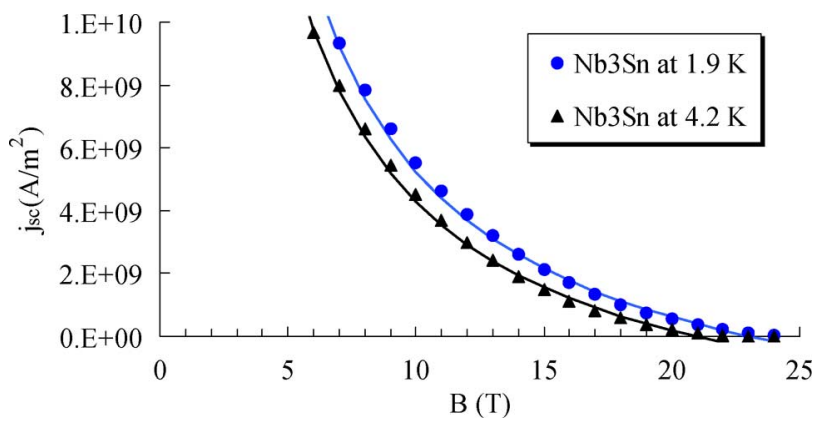

Fig. 7. Critical surface of the $\mathrm{Nb}_{3} \mathrm{Sn}$ according to Summer fit (markers) and proposed fit of Eq. (15) (solid lines).

\section{CRITICAl GRADIENT FOR Nb-Ti-Ta}

Even though the superconducting properties of the Nb-Ti-Ta compound have been studied since several years, no applications to magnets have been carried out yet. The critical surface of the $\mathrm{Nb}$-Ti-Ta has a similar slope to the $\mathrm{Nb}$-Ti, and a higher critical field of about $1.5 \mathrm{~T}$. Therefore the previous results can be simply generalized to $\mathrm{Nb}-\mathrm{Ti}$-Ta by adding about $10 \%$.

\section{CRITICAL GRAdient FOR $\mathrm{Nb}_{3} \mathrm{Sn}$}

The critical surface of the $\mathrm{Nb}_{3} \mathrm{Sn}$ is not linear over our domain of interest, and therefore more complicated approximations must be used. The Kramer law [8] (and the related Summer fit [9]) is the most commonly used; unfortunately its algebraic form does not allow an explicit solution for the critical gradient as done in (10) for the linear case of Nb-Ti. Here we propose a simple hyperbolic fit

$$
j_{s c}=c\left(\frac{b}{B}-1\right) \quad B<b .
$$

We set $\mathrm{c}=4 \times 10^{9} \mathrm{~A} / \mathrm{m}^{2}$ and $b=23.1 \mathrm{~T}$ at $1.9 \mathrm{~K}$ and $\mathrm{c}=3.9 \times 10^{9} \mathrm{~A} / \mathrm{m}^{2}$ and $b=21 \mathrm{~T}$ at $4.2 \mathrm{~K}$ : these values between $11 \mathrm{~T}$ and $17 \mathrm{~T}$ agree within 5\% with the Kramer law using the typical parameters for a good $\mathrm{Nb}_{3} \mathrm{Sn}$ superconductor, namely having $j_{\mathrm{sc}}=3 \times 10^{9} \mathrm{~A} / \mathrm{m}^{2}$ at $12 \mathrm{~T}$ and $4.2 \mathrm{~K}$ (see Fig. 7). This simple approximation has the advantage of having an explicit solution for the critical gradient:

$$
G_{c}=\frac{\kappa c \gamma}{2}\left(\sqrt{\frac{4 b}{\beta \kappa c}+1}-1\right)
$$

and substituting the lay-out parameters ((3)-(6)) one obtains an explicit equation for the $\mathrm{Nb}_{3} \mathrm{Sn}$ case. As for $\mathrm{Nb}-\mathrm{Ti}$, the maximum with respect to $w$ provides the estimate of the higher critical gradient reachable for a given aperture. Results are given in Fig. 8: $r G$ ranges from $13 \mathrm{~T}(r=30-40 \mathrm{~mm})$ to $16 \mathrm{~T}$ $(r=100 \mathrm{~mm})$, and is much smaller than the critical field (nearly a factor two). This is due to the shape of the critical surface. For a fixed aperture radius of $r=35 \mathrm{~mm}, \mathrm{Nb}_{3} \mathrm{Sn}$ provides a critical gradient that is up to $50 \%$ larger than $\mathrm{Nb}$-Ti. For a fixed critical gradient of $200 \mathrm{~T} / \mathrm{m}, \mathrm{Nb}_{3} \mathrm{Sn}$ quadrupoles have an aperture that is up to $70 \%$ larger than $\mathrm{Nb}-\mathrm{Ti}$.

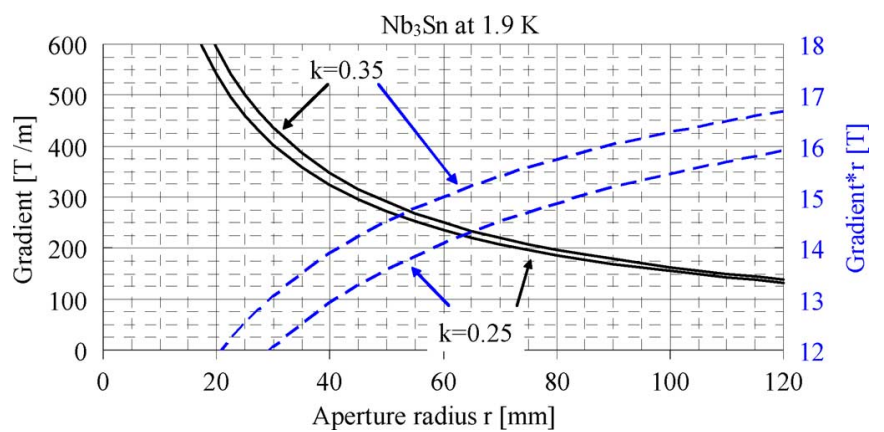

Fig. 8. Maximum critical gradient (solid line) and maximum critical gradient times aperture (dashed line) versus aperture radius for a sector coil of $\mathrm{Nb}_{3} \mathrm{Sn}$ at $1.9 \mathrm{~K}$ for two different filling factor values.

\section{CONCLUSION}

We derived a formula giving the critical gradient as a function of the superconducting properties of the material, of the magnet aperture, and of the quantity of cable used in the cross-section for the lay-out shown in Fig. 1. This formula agrees within 3.5\% with results relative to 10 non-graded magnets, and cases with grading give $4-8 \%$ more.

The formula can be used to derive the maximum critical gradient reachable with a superconducting quadrupole of a given aperture. One finds that the gradient times the aperture radius is much smaller than the naïve limit given by the critical field of the material. The loss becomes more and more relevant for smaller apertures: for $r=10 \mathrm{~mm}$ one obtains values that are $1 / 2$ the naïve limit for $\mathrm{Nb}$-Ti. The effect is stronger for $\mathrm{Nb}_{3} \mathrm{Sn}$. This suggests that quadrupoles with very small apertures do not exploit well the potential of superconducting material. It remains an open issue whether a coil could be designed to provide better performance than our model: indeed, all the analyzed designs agree with our model.

For aperture radii of the order of $35 \mathrm{~mm}$, as in the Large Hadron Collider interaction regions, the aperture radius times the gradient is at most $9.5 \mathrm{~T}$ for the $\mathrm{Nb}-\mathrm{Ti}$ and $14 \mathrm{~T}$ for the $\mathrm{Nb}_{3} \mathrm{Sn}$ at $1.9 \mathrm{~K}$. Graded coil, which are not considered in our model, can give 5\%-10\% more.

\section{REFERENCES}

[1] H. Brechna, Superconducting Magnet Systems. Berlin: Springer Verlag, 1973

[2] M. N. Wilson, Superconducting Magnets. Oxford: Clarendon Press, 1983.

[3] L. Rossi, "The LHC main dipoles and quadrupoles towards series production," IEEE Trans. Appl. Supercond., vol. 12, pp. 219-227, 2002.

[4] G. Ambrosio et al., Preliminary Proposal of a $\mathrm{Nb}_{3} \mathrm{Sn}$ Quadrupole Model for the Low- $\beta$ Insertions of the LHC INFN TC-95-25, 1995.

[5] S. Caspi and P. Ferracin, "Limits of $\mathrm{Nb}_{3} \mathrm{Sn}$ accelerator magnets," in Proc. PAC 2005 [Online]. Available: http://www.jacow.org

[6] S. Caspi, P. Ferracin, and S. Gourlay, "Graded high field Nb3Sn dipole magnets," in 19th Magnet Technology Conference, IEEE Trans. Appl. Supercond., 2006, in press.

[7] E. Todesco and L. Rossi, "Electromagnetic design of superconducting quadrupoles," Phys. Rev. STAB, vol. 9, p. 102401, 2006.

[8] E. J. Kramer, "Scaling laws for flux pinning in hard superconductors," J. Appl. Phys., vol. 44, pp. 1360-1370, 1973.

[9] L. Summers et al., "A model for the prediction of $\mathrm{Nb}_{3} \mathrm{Sn}$ critical current as a fucntion of field, temperature, strain and radiation damage," IEEE Trans. Appl. Supercond, vol. 27, pp. 2041-2044, 1991. 